\title{
Haptic Interaction and Interactive Simulation in an AR Environment for Aesthetic Product Design
}

\author{
Monica Bordegoni, Francesco Ferrise, and Marco Ambrogio \\ Politecnico di Milano, Dipartimento di Meccanica, \\ Via G. La Masa 34, 20156 Milano, Italy \\ \{monica.bordegoni@, francesco.ferrise@, \\ marco.ambrogio@mail.\}polimi.it
}

\begin{abstract}
Market rules show that most of the times the aesthetic impact of a product is an important aspect that makes the difference in terms of success among different products. The product shape is generally created and represented during the conceptual phase of the product and the last trends show that the use of haptic devices allows users to more naturally and effectively interact with 3D models. Nevertheless the shape needs to satisfy some engineering requirements, and its aesthetic and functional analysis requires the collaboration and synchronization of activities performed by various experts having different competences and roles. This paper presents the description of an environment named PUODARSI that allows designers to modify the shape of a product and engineers to evaluate in real-time the impact of these changes on the structural and fluid dynamic properties of the product, describing the choice of the software tools, the implementation and some usability tests.
\end{abstract}

Keywords: Mixed reality, haptic interaction, interactive simulation.

\section{Introduction}

The trends of the market suggest that a lot of importance in the whole Product Development Process (PDP) must be given to the definition of the aesthetical properties of the shape. The same product must be also efficient from the technological point of view and so requires some engineering tests to be done on it in order to be optimized. These requirements generate a process that can be imagined like a closed loop among designers that define the shape and engineers that analyze it. Despite the availability of new technologies like those of Virtual and Augmented Reality that can be considered as tools that enable a 'natural' interaction with computer generated worlds, the PDP in the world of industry is still based on old techniques and technologies that are considered robust and reliable. The designers use to work on physical prototypes and this is because some of them are manually skilled, or sometimes are used to define their shapes sketching on two dimensional papers. There are also CAD systems that help them to define computer geometrical models but they are still based on human computer interfaces like mouse and keyboards that often do not allow the designer to fully express his idea of shape and are not integrated with VR devices. The use of the CAD systems allows to get immediately, as a result of the modeling phase, a prototype that is more flexible than the physical ones because can be used some tests and evaluations. 
Regarding the engineering tests, the algorithms based on the Finite Element Method are helping to substitute the tests made in the past on physical prototypes. Some examples of this can be found in the Computational Fluid Dynamics analysis, known as CFD that is substituting the wind tunnel based analysis.

The integration of Virtual and Augmented Reality Technologies together with the use of virtual prototypes in the PDP and in particular in the phases when the shape is defined and analyzed would allow to reduce the time to market.

The paper describes a research carried out in the contest of the PDP with the aim of changing the way of defining a shape and of analyzing it, making it more easy and intuitive thanks to the use of VR/AR techniques and technologies together with some real-time analyses. In the first part a brief state of the art is presented, first on the VR/AR technologies and their usage in the contest of PDP and of the algorithms that enable the interactive simulations. The paper then describes how the classical process of shape modeling and engineering simulation can be virtualized, with issues and potentialities, and the solutions adopted in the contest of the PUODARSI project. So the paper describes the system that has been developed in order to prove the concept and run some usability tests. Then the conclusions are presented.

\section{State of the Art of Haptic, AR Devices and Interactive Simulations in the Design Domain}

There are some examples of the use of Virtual and Augmented Reality technologies supporting the PDP. Most of these applications are focused on the design review phase, i.e. when the product has been defined and just small changes can be taken. Our research aims at introducing the use of these technologies already in the preliminary phases of the PDP, i.e. in the conceptual and embodiment phases, where the product is still in its development phase. In the following a brief state of the art of the interested technologies is presented with regard to their application in the PDP.

\subsection{Haptic Technologies}

Haptic Interaction is meant as a way to add the sense of touch to Virtual Environments. There are many devices on the market, but basically point based and with three or more degrees of freedom [1]. The devices basically differ in terms of degrees of freedom in input and output, the work volume, and strength of the returned force. Among these devices the most used are the Phantom Sensable products [2], the Haptic Master-MOOG FCS [3], the devices of Force Dimension [4], and those distributed by Haption [5].

In the field of research some devices that return a linear contact-type [6] or surface contact-type with the virtual object have been developed, but at the time they're still prototypes. Haptic devices are generally provided with proprietary library for the development of applications, enabling in an easy way to create haptic environments, but with very low level visualization environments. However, there are some types of open source libraries, already integrated with high level visualization libraries and sound rendering and also algorithms for the creation of physics-based environments, 
which permit the development of applications regardless of device used. Examples of such libraries are CHAI3D [7], H3D [8], HaptikLibrary [9] and OpenSceneGraph Haptic Library [10].

In the specific area of design and product development there are a variety of applications ranging from the Virtual Sculpting commercial package FreeForm distributed by Sensable [2], which is the first application based on a physics-based interaction with a clay modeling tool, going up to the application that allows the Virtual Assembly in CATIA, a commercial CAD system, distributed by Dassault Systemes. Finally there are a number of research projects in which the haptic interaction is used in the creation of forms [11] by virtually replicating the behavior of some tools used by designers in the creation of forms from clay models, like the rake or the sanding tool. There's another example of a linear haptic device in a multimodal environment used as a means to return to the contact a characteristic line of a style surface in order to enable the qualitative assessment and modification of the same [12].

\subsection{Augmented Reality Technologies}

Augmented Reality (AR) allows to contextualize a Virtual Prototype in a real environment i.e. giving the opportunity to the user to see a digital model into a real scene. Nowadays computer graphics technology in fact is able to generate a photorealistic representation of the digital model but a good contextualization of the VP represents a challenge yet. The simplest architecture to realize an AR environment is composed by a camera and a monitor; the camera acquires the real scene then the image acquired is processed by the pc that merges digital model in the real environment.

In order to superimpose the virtual object in the real scene it is fundamental the use of a tracking system that provides the correct position and orientation of the virtual object respect to the user's point of view. The pioneer tracking system for AR is certainly represented by ARToolkit [13] library. This is a very simple tracking system that, by processing the camera video streaming, derives position and orientation of the virtual object by a 2D marker. Starting from this idea many other tracking libraries $[14,15]$ have been developed to spending much effort to the marker-less tracking system and to improving precision and accuracy. Regarding the use of AR applications for PDP, in these years many research groups developed AR applications in order to improve, speed and simplify the different product development phases. Friedrich [16] describes a testing of AR application in development, production, and servicing of the product in use or the machines and systems required for service and production. Doil et al. [17] describes potential benefits and development of a prototypical AR-System for the support of manufacturing planning tasks. Santos et al [18] describes an application for collaborative mobile design review that allow to simplify the review procedures and to reduce the number of physical prototypes that are realized for review phase. Another interesting application is related to the exploitation of results of numerical simulation data. In [19] is presented a framework that allows to see the results of a CFD analysis directly on the physical prototype of the product in the developing phase by using a tablet pc. This framework is still used in the contest of the project described in this paper. 


\subsection{Interactive Simulation in CFD Analysis}

In the PDP, numerical analyses are always used to verify and validate the virtual model of the product. The most used commercial software in industrial field for CFD analyses is Fluent, recently purchased by Ansys Inc. [20]. Moreover a number of open-source algorithms allow us to perform high accurate structural and CFD analyses and are widely used in the research field due to the fact that they are completely open and extensible. Regarding the CFD analysis we find, for example, OpenFOAM [21] and OpenFlower [22]. The analysis process is usually divided into three steps: pre-processing, analysis and post-processing. The first step consists of the discretization of the physic problem subject of the analysis, generally the geometric model and the control volumes around it for CFD analyses, and the definition of the initial conditions for the analysis. In the second step the software runs the numerical analysis and creates the results data. The third and last step represents the review of the output data obtained from analysis, which can be velocity and pressure distribution for CFD analyses. Generally, in the analysis phase of a product, a lot of time is spent by engineers, especially to correctly define the pre-processing parameters, but also to perform the analysis and find the solution of the problem, due to slow resolution algorithms developed in commercial software. Many studies have been done in order to speed-up the simulations, especially concerning the case of the simulation of the behavior of fluids, but all of these algorithms have been developed for Computer Graphics purposes. These algorithms, also if not suitable for pure engineering problems that sometimes need a high accuracy of results, are based on physical models and so are useful when, as in our research, the aim is to find a way to reduce the computational time of the analysis phase and the accuracy of the results is not the most important goal. Fournier and Reeves [23] describe a simple model to simulate the behavior of the surface of the Ocean that is based on Rankine model, where particles of water describe circular or elliptical orbits. Chen and Lobo define a new method [24] to perform in real-time three-dimensional CFD simulations in a dynamic virtual environment with moving obstacles. In the last years considerable improvements have been reached in the field of real-time fluid simulations, due to new programming technologies and different definitions of physical model of fluids. In particular Muller and Gross [25] describe a new method to simulate fluids with free surfaces, using Smoothed Particle Hydrodynamics. An example of fast engineering analyses based on classical CFD algorithms has been developed in the context of a research project where an engineering CFD tool has been optimized in order to perform fast analyses, connected to the change of the shape of style object [26], and the results show that fast analysis is possible if the phase of the creation of the mesh is optimized and the initial conditions are kept constant.

\section{Issues in Virtualizing Some Real Tasks in the PUODARSI Solution}

This section describes the implementation of the concepts highlighted above in particular presenting the PUODARSI solutions. The whole system is complicated and made of simpler and standalone modules, each for one purpose, and especially 
implemented in order to be reusable as additional tools for other existing applications. So all the modules share the same file format and communicate through server/client connections. Engineers and Designers make use of different representations and different language in order to express their concepts. In order to make them collaborate and work together, as will be described, an annotation tool has been added to the system.

\subsection{Modification of the Shape}

The Haptic module has been implemented in H3D. The module takes as input a STL file format and translates it in a X3D model. Then this object can be managed with a three-dimensional Gaussian modification tool where the user can choose some parameters like the width of the tool, and the softness of the material that he's managing. Every time that the model is modified, the system stores it in the RAM memory, in order to allow the undo/redo operations. When the desired shape is obtained then the designer freezes the model that is saved in a new file named VerN.stl where $\mathrm{N}$ is the number of times the file is saved, and sends it to the shared folder with the analysis tools. Figure 1 shows the user that is deforming a shape using a Sensable Phantom Omni device, in a Sensegraphics workbench.

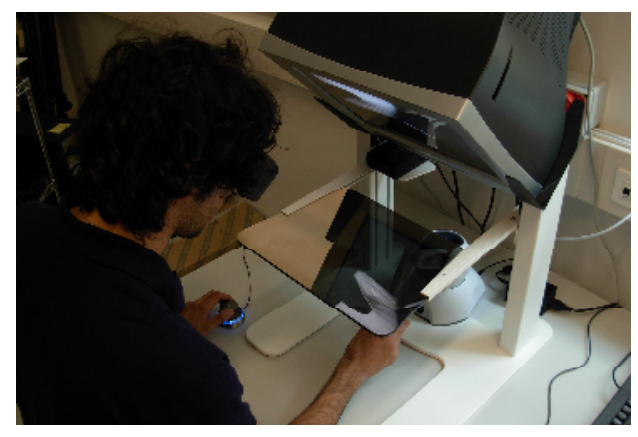

Fig. 1. Example of the haptic modeling tool used for the modification phase

\subsection{Simulation and Results}

The simulation module has been defined taking into account problems regarding preprocessing step, simulation time, visualization and analysis of post-processing data. The implementation of the module for CFD analysis has been done using the open source OpenFOAM library, a set of different tools used to simulate specific problems in engineering mechanics, including computational fluid-dynamic problems. The choice of this library is attributable to the big amount of tools that can easily solve compatibility problems with the other modules, and also to the time performances of the solvers integrated in the library.

In order to share geometric models with the haptic module, it has been necessary to use tessellated surfaces, define a control volume around them and then create a three dimensional mesh on the volume obtained by the subtraction of the volume of the model from the control volume. This step has been done defining a $\mathrm{C}++$ code to 

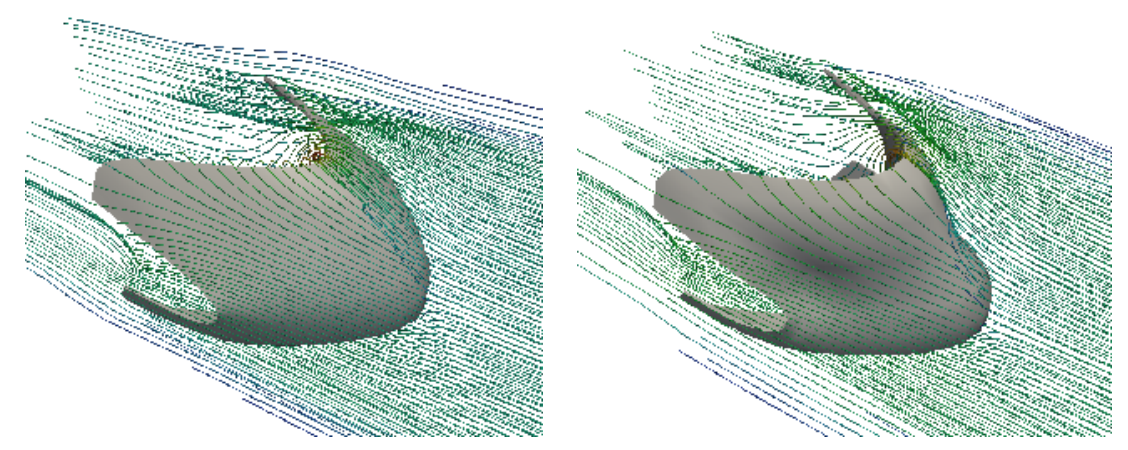

Fig. 2. CFD simulation on a windscreen before and after the modification

import tessellated geometries (STL files) and create the control volume in the software GMSH, used to generate the mesh of elements. To increase the velocity of the analysis, the initial conditions, like inlet velocity and outlet pressure, are automatically assigned to the corresponding surfaces of the control volume; in particular an inlet velocity of $30 \mathrm{~m} / \mathrm{s}$ and atmospheric pressure are chosen for the analyses. Once chosen the geometry to analyze, the simulation module automatically runs all the steps described before and consequently launches the analysis.

When the analysis is complete, the results are sent to the Visualization System that renders these data. The results of the analysis are transformed into a format that is compatible with VTK. The Visualization Toolkit - VTK is an open source library for Scientific Visualization that allows developers to easily visualize data obtained from scientific simulations.

Figure 2 shows the results of the shape modification on the CFD analysis of the windscreen model.

\subsection{Annotation Tool in AR and Exchange of Information}

The annotation tool has been implemented in order to allow the exchange of information among the different users and has been connected to a Database that stores all the information of the PDP in order to allow a reuse of them. The annotation component has been developed in C++ using QT libraries. The idea was to create a VTK widget able to visualize only the visual links related to the annotations connected to the models, or the CFD/FEM analysis. Instead the annotation component has been implemented by means of a QT dialog box using which the user can create or manage annotations. In this way it is possible to exploit the QT library for defining the textediting module and for integrating components to manage the threads of notes. Moreover QT supports easy integration of different multimedia files like audio, video or pictures related to the annotation. Both the VTK component and QT component access archives storing 3D models, analysis and annotations through a KM module developed to guarantee a transparent information retrieval with respect to technical details of the databases. The VTK objects are used then in the VTK4AR framework [19] that allows us to view and exploit the results in an intuitive way. Figure 3 shows an example of the annotation module in AR. 


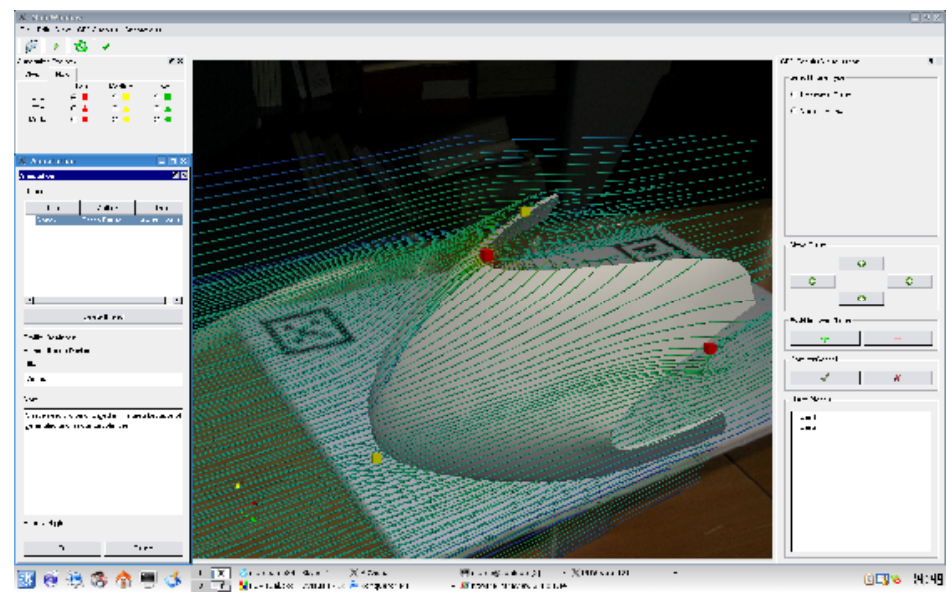

Fig. 3. Screenshot of the AR annotation tool, representing the results of the CFD analysis on the original model

\section{Testing and Results}

A prototype of the system has been developed in order to perform some usability tests and to get the impressions of the final user about the effectiveness of the approach. In the following a brief description of the testing phases and of the results is presented.

\subsection{Description of the Usability Tests}

The tests of the system have been articulated as following: ten users, five designers and five engineers had to perform the following tasks: 1) the designers were asked to change the shape of the windscreen as illustrated in the Figure 2;2) the engineers were asked to verify the results on the CFD analyses in the AR environment; 3) engineers had to put some annotations on the geometry and on the streamlines in the AR environment and the designers had to read and add other notes. At the end of these tasks the users were asked to answer to some questions regarding the usability of the system for each task performed; the questions concerned their general impressions, knowledge acquisition, functionalities divided into systems components and GUI layout, goal achievements. The results are reported in the following section.

\subsection{Results}

After using the system, the testers were asked to answer to a questionnaire. Figure 3 reports the answers to the questions provided by the testers, scoring each question from 0 to 6 . The general users' impressions about the system show that despite they like the concept and the innovation of the whole system, there is still some work to do on each module in order to reach a really usable system. From the data reported on the chart of Figure 3 it is possible to notice that the users perceive that the system can help to reduce the time needed to create and test the model, but they judge not so 


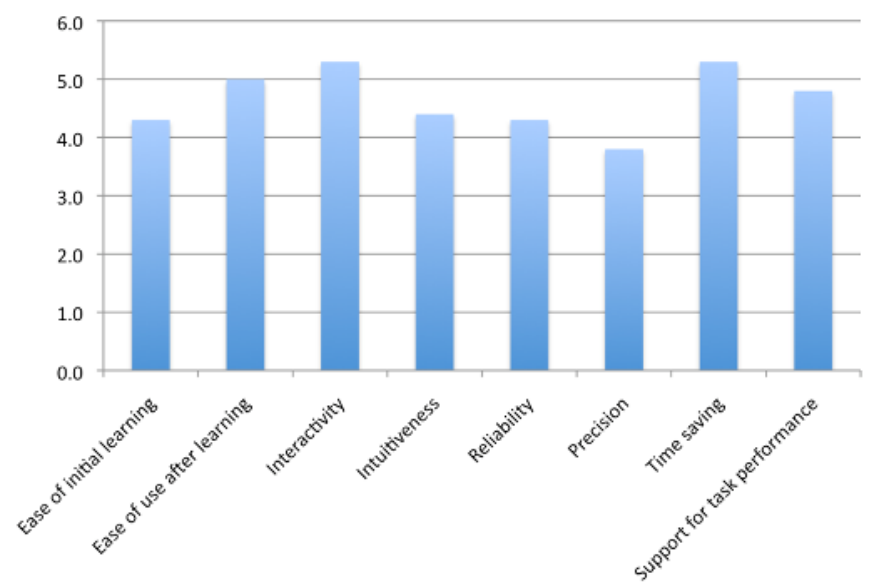

Fig. 4. Results of the general impressions of the users during the usability tests

efficient the precision and the reliability of the system. Then, the users state that the system is not so easy to use at the beginning of the tasks but after a period of learning it may become a good support for tasks performances thanks of the new kind of interaction implemented.

\section{Conclusions}

The paper described a contribution at the product development process innovation based on the use of VR and AR devices together with interactive simulation. The aim was to create a simulation aided design, and to bring closer the worlds of engineers and designers. A prototype has been build in order to test the effectiveness, to prove the concept and to get some initial feedbacks from the final users.

The tests show that far from being usable by the industrial world, the PUODARSI framework introduces some single innovations that if developed and exploited can become part of whole process. For example the users like the way of interacting with models thanks to the haptic or AR devices and this is because they consider the interaction more intuitive, but what they judge very important is the possibility to reduce the time needed to realize and test the product thanks to the collaboration of designers and engineers and this is mainly due to the reduction of the time for analyses and the exchange of information through the annotation system. So the next developments of the system will aim at introducing some adjustments at the interaction but also at the precision and reliability of the system.

Acknowledgments. Part of the research described in the paper has been developed in the context of the PUODARSI (Product User Oriented Development based on Augmented Reality and interactive SImulation) project (http://www.kaemart.it/puodarsi), partially funded by the Italian Ministry of University and Research. 


\section{References}

1. Hayward, V., Ashley, O., Hernandez, M.C., Grant, D., RoblesDeLaTorre, G.: Haptic interfaces and devices. Sensor Review 24(1), 16-29 (2004)

2. PHANToM device, SenSable Technologies Inc., http: / /www. sensable.com

3. FCS-HapticMaster, MOOG-FCS, http: / / www. moogfcs.com/robotics

4. Force Dimension Haptic devices, http: / / www. forcedimension.com/

5. Virtuose, Haption, http://www. haption. com/

6. Bordegoni, M., Cugini, U., Covarrubias, M.: Design of a visualization system integrated with haptic interfaces. In: Horvath, I., Rusak, Z. (eds.) Proocedings of the TMCE (2008)

7. CHAI3D Haptic Library, http: / /www. chai3d.org/

8. H3D Haptic Library, http: / / www. h3d.org/

9. Haptik Library, http: / /www . haptiklibrary. org /

10. OpenSceneGraph Haptic Library, http://sourceforge.net/projects/osghaptics

11. Bordegoni, M., Cugini, U.: Haptic modeling in the conceptual phases of product design. Virtual Reality Journal 9(1), 192-202 (2006)

12. Bordegoni, M., Ferrise, F., Shelley, S., Alonso, M., Hermes, D.: Sound and tangible interface for shape evaluation and modification. In: Proceeding of HAVE 2008 - IEEE International Workshop on Haptic Audio Visual Environments and their Applications (2008)

13. Kato, H., Billinghurst, M.: Marker tracking and hmd calibration for a video-based augmented reality conferencing system. In: International Workshop on Augmented Reality, p. 85 (1999)

14. Fiala, M.: Artag, a fiducial marker system using digital techniques. In: IEEE Computer Society Conference on Computer Vision and Pattern Recognition, CVPR 2005, June 2, 2005, vol. 2, pp. 590-596 (2005)

15. Schmalstieg, D., Fuhrmann, A., Hesina, G., Szalavari, Z., Encarnacao, L.M., Gervautz, M., Purgathofer, W.: The studierstube augmented reality project. Tech. Rep. TR-186-2-00-22, Institute of Computer Graphics and Algorithms, Vienna University of Technology (2000)

16. Wohlgemuth, W., Triebfürst, G.: Arvika: augmented reality for development, production and service. In: Proceedings of DARE 2000 on Designing augmented reality environments, pp. 151-152. ACM Press, New York (2000)

17. Doil, F., Schreiber, W., Alt, T., Patron, C.: Augmented reality for manufacturing planning. In: EGVE 2003: Proceedings of the workshop on Virtual environments 2003, pp. 71-76. ACM Press, New York (2003)

18. Santos, P., Stork, A., Gierlinger, T., Pagani, A., Paloc, C., Barandarian, I., Conti, G., de Amicis, R., Witzel, M., Machui, O., Jimanez, J., Araujo, B., Jorge, J., Bodammer, G.: Improve: An innovative application for collaborative mobile mixed reality design review. International Journal on Interactive Design and Manufacturing 1(2), 115-126 (2007)

19. Bruno, F., Caruso, F., Ferrise, F., Muzzupappa, M.: Vtk4ar: An object oriented framework for scientific visualization of cae data in augmented reality. In: Gallo, G., Battiato, S., Stanco, F. (eds.) Proceedings of Eurographics Italian Chapter Conference, Eurographics, pp. 76-81 (2006)

20. Fluent, by Ansys Inc., http: / /www. fluent. com/

21. OpenFOAM: The Open Source CFD Toolbox, http: / /www. opencfd.co.uk/ 
22. OpenFlower, http: //openflower. sourceforge.net/

23. Fournier, A., Reeves, W.T.: A simple model of ocean waves. SIGGRAPH Comput. Graph. 20(4), 75-84 (1986)

24. Chen, J.X., da Vitoria Lobo, N., Hughes, C.E., Moshell, J.M.: Real-time fluid simulation in a dynamic virtual environment. IEEE Computer Graphics and Applications 17(3), 52 $61(1997)$

25. Muller, M., Charypar, D., Gross, M.: Particle-based fluid simulation for interactive applications. In: Proceedings of the 2003 ACM SIGGRAPH/Eurographics symposium on Computer animation, Eurographics Association, pp. 154-159 (2003)

26. Bordegoni, M., Ferrise, F., Ambrogio, M., Bruno, F., Caruso, F.: A multi-layered modelling architecture for virtual design. In: Proceedings of IDMME Virtual Concept 2008 Conference (2008) 